INPLASY

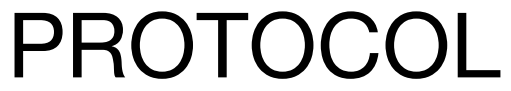

To cite: Zheng et al. Non Pharmacological and Noninvasive Interventions for Labor:

A Network Meta-analysis. Inplasy protocol 202070081. doi:

10.37766/inplasy2020.7.0081

Received: 18 July 2020

Published: 18 July 2020

Corresponding author: Jinxuan Zheng

woshizjx512@163.com

Author Affiliation:

Dongguan maternal and child health care hospital, Yichang Central People's Hospital

Support: None.

Review Stage at time of this submission: The review has not yet started.

Conflicts of interest: None.

\section{Non Pharmacological and Non- invasive Interventions for Labor: A Network Meta-analysis}

Zheng, J1; Li, Z2; Zhou, X³.

Review question / Objective: P:Primipara with single pregnancy is not limited to nationality and race. I: (1) Hypnosis (2) Aromatherapy (3) Immersion in water (4) Relaxation Techniques (5) T ranscutaneous electrical nerve stimulation (6) Manual methods C:Placebo is not treated or different interventions are compared. O: Main outcome indicator: (1) Analgesic effect; (2) Labor time Time of first stage, second stage, third stage and total stage. Secondary Outcome Indicator (1) Delivery method Cesarean birth rate, device assisted birth rate, natural delivery rate (2) Apgar score of newborn Use the Apgar score scale to assess the Apgar score at $1 \mathrm{~min}$ and $5 \mathrm{~min}$ after birth (3) Adverse pregnancy outcomes (1) Maternity Postpartum bleeding volume; Perineal trauma (three or four levels of tearing); Postpartum infection rate; Postpartum mortality (2)Newborn Neonatal asphyxia rate; Neonatal infection rate, including infection markers such as fever and increased white blood cell count.

Condition being studied: The organization has access to various databases, team members have registered and published meta-analysis, and are familiar with the process, etc

INPLASY registration number: This protocol was registered with the International Platform of Registered Systematic Review and Meta-Analysis Protocols (INPLASY) on 18 July 2020 and was last updated on 18 July 2020 (registration number INPLASY202070081).

\section{INTRODUCTION}

Review question / Objective: P:Primipara with single pregnancy is not limited to nationality and race. I: (1) Hypnosis (2)
Aromatherapy (3) Immersion in water

(4) Relaxation Techniques (5) T ranscutaneous electrical nerve stimulation

(6) Manual methods C:Placebo is not treated or different interventions are 
compared. O: Main outcome indicator: (1) Analgesic effect; (2) Labor time Time of first stage, second stage, third stage and total stage. Secondary Outcome Indicator (1) Delivery method Cesarean birth rate, device assisted birth rate, natural delivery rate (2) Apgar score of newborn Use the Apgar score scale to assess the Apgar score at $1 \mathrm{~min}$ and $5 \mathrm{~min}$ after birth (3) Adverse pregnancy outcomes (1) Maternity Postpartum bleeding volume; Perineal trauma (three or four levels of tearing); Postpartum infection rate; Postpartum mortality (2)Newborn Neonatal asphyxia rate; Neonatal infection rate, including infection markers such as fever and increased white blood cell count.

Condition being studied: The organization has access to various databases, team members have registered and published meta-analysis, and are familiar with the process, etc.

\section{METHODS}

Search strategy: Search terms: (1) Hypnosis, Mesmerism, Hypnoses; (2) Aromatherapy, Aroma Therapy, Aroma Therapies, Aromatherapies, Therapies, Aroma; (3) Therapies (4)Relaxation, yoga, Muscle Stretching Exercises, Music, Musics, Audio, Audio-Visual Media, Audiovisual Media (5) $T$ ranscutaneous electrical nerve stimulation, Analgesic Cutaneous Electrostimulation, Electric Stimulation, Transcutaneous. Electroanalgesia, Percutaneous Electric Nerve Stimulation, TENS, Transdermal Electrostimulation, Electrical Stimulation, Transcutaneous, Percutaneous Electrical Nerve Stimulation, Transcutaneous Electrical Nerve Stimulation, Transcutaneous Nerve Stimulation, Cutaneous Electrostimulation, Analgesic, Electrostimulation, Analgesic Cutaneous, Electrostimulation, Transdermal, Nerve Stimulation, Transcutaneous, Stimulation, Transcutaneous Electric, Stimulation, Transcutaneous Electrical, Stimulation, Transcutaneous Nerve, Transcutaneous Electric Stimulation, Transcutaneous Electrical Stimulation; (6) Massage,
Reflexology, Zone Therapy, Bodywork, Craniosacral Massage, Rolfing, Therapy, Zone, Bodyworks, Massage, Craniosacral, Therapies, Zone, Zone Therapies; (7) Birth, childbirth, births, childbirths and parturition. In each group, the title words and the text words are connected by "or" and use "and" to search with the other two groups of words.

Participant or population: Primipara with single pregnancy is not limited to nationality and race.

Intervention: (1)Hypnosis (Mesmerism; Hypnoses) mainly through training to correct the belief of childbirth pain deeply rooted in the maternal brain, change the concept of childbirth pain into childbirth comfort, adjust the mentality, change the thinking, and treat the inner world. (2)Aromatherapy (Aroma Therapy Aroma Therapies、Aromatherapies、Therapies, Aroma) When you are giving birth, add the massage or spray of essential oil, so that the fragrance of essential oil can be filled with the whole delivery room, and create a warm environment to relieve tension and pressure, and gradually relax the body of the lying in woman. In this case, the pain of the mother will also be reduced, and the time for production will be greatly reduced. (3)Immersion in water In water deliveries, the signals produced by warm water stimulate the skin of the baby to pass through the fast fibers, which can block or reduce the pain signal to the brain, and reduce the pain of the brain. At the same time, it is conducive to relaxing the muscles of the puerpera, and then is conducive to the expansion of the uterine orifice and to reduce the laceration of the birth canal. (4)Relaxation Techniques (Relaxation Technics、Therapy, Relaxation; yoga, Muscle Stretching Exercise; Music、 Musics.

Comparator: Placebo is not treated or different interventions are compared.

Study designs to be included: Randomized controlled trial (RCTs). 
Eligibility criteria: (1) gestational age was more than 37 weeks; (2) intervention measures: the experimental group adopted a single non drug labor analgesia intervention measure; (3) there was a comparability between the non drug delivery group and the control group in terms of demographic variables.

Information sources: Database: PubMed, Cochrane Library, EMBASE, CNKI, Wan Fang, VIP, etc.

Main outcome(s): (1) Analgesic effect The visual analogue scale (VAS) score was used to assess the degree of maternal pain. (2) Labor time Time of first stage, second stage, third stage and total stage.

Additional outcome(s): (1) Delivery method Cesarean birth rate, device assisted birth rate, natural delivery rate (2) Apgar score of newborn Use the Apgar score scale to assess the Apgar score at $1 \mathrm{~min}$ and $5 \mathrm{~min}$ after birth (3) Adverse pregnancy outcomes

(1) Maternity Postpartum bleeding volume; Perineal trauma (three or four levels of tearing); Postpartum infection rate; Postpartum mortality (2)Newborn Neonatal asphyxia rate; Neonatal infection rate, including infection markers such as fever and increased white blood cell count.

Quality assessment / Risk of bias analysis: The Cochrane risk bias assessment tool was used to evaluate the seven items of each original document: (1) the generation of random sequences (selection bias); (2) blind distribution (selection bias); (3) all participants and personnel used blindness (Executive bias); (4) blind evaluation of results (observation bias);Other items. Each item is classified into three categories: low risk, high risk and unclear risk. The recommended level of each document is: A: low bias, that is, it satisfies 4 items or more, and the possibility of bias is small. B level: moderate bias, that is, 2 or 3 items are fully met, with moderate possibility of bias; C grade: high bias.Totally meet 1 items or one is not satisfied, there is a high likelihood of bias. Finally, the text, form or graphic method is used to show the evaluation results of all the papers.

Strategy of data synthesis: Traditional meta-analysis Meta-analysis of data using RevMan5.3 software, Stata15.0 software and TSA 0.9 software, binary classification data select oddsratio (OR) and $95 \%$ confidence interval $(\mathrm{Cl})$ as meta-analysis Combined effect indicators; continuous data, if the dimension is consistent, select the weighted mean difference (MD) and $95 \% \mathrm{Cl}$ as the effect indicator, if the measurement method is different, choose the standardized mean difference (SMD) and $95 \% \mathrm{Cl}$ It is an effect indicator. Use sensitivity analysis to eliminate the included studies one by one, and reconsolidate the observation statistics to evaluate the stability of the conclusions. Determine the heterogeneity between studies by 2 test and 12 quantitative analysis, if $P>0.1,12<50 \%$ think that the heterogeneity between studies is acceptable, choose a fixed effect model for Meta analysis; if $\mathbf{P} \mathbf{5 0 \%}$ think that the heterogeneity between studies is large, choose a random effect model for Meta analysis when merging, and pass Subgroup analysis to find the source of heterogeneity. Use funnel chart and Egger's test to determine whether there is a potential publication bias. Then, use TSA0.9 software to conduct sequential analysis of the test to verify the accuracy of the meta analysis results. network meta-analysis First use the stata 15 software to draw the network diagram, and then use the Gemtc 14.3 software to perform the mesh meta analysis. Regarding the model selection, the point-split model is used for the inconsistency test. If there is no statistical difference $(P>0.05)$, the consistency is used. The model is used for analysis; otherwise, the inconsistent model is used for analysis. Counting data is expressed by odds ratio (OR), and measurement data uses mean difference (MD) or standardized mean difference (SMD) as the effect amount, and the above results all give $95 \%$ Confidence Interval (Cl); use the $\mathbf{Q}$ test for hypothesis testing, expressed. 
Subgroup analysis: When the heterogeneity combined by traditional meta-analysis is high, use subgroup analysis to find the source of heterogeneity. The set groups are as follows: intervention time, number of interventions, intervention group age (median), publication year, and quality of the included study, The characteristics of the participating population, etc.

Sensibility analysis: None.

Language: Chinese and English.

Country(ies) involved: China.

Keywords: Non Pharmacological and Noninvasive Interventions, Labor, A Network Meta-analysis.

Contributions of each author:

Author 1 - Jinxuan Zheng.

Author 2 - Zifeng Li.

Author 3 - Xiaojing Zhou. 